\title{
Light Element Imaging Technique at Low Dose Condition by Processing Simultaneously Obtained STEM Images Using a Segmented Detector
}

\author{
Kousuke Ooe ${ }^{1}$, Takehito Seki ${ }^{1}$, Yuichi Ikuhara ${ }^{1,2}$ and Naoya Shibata ${ }^{1,2}$ \\ 1. Institute of Engineering Innovation, School of Engineering, the University of Tokyo, Tokyo, Japan. \\ 2. Nanostructure Research Laboratory, Japan Fine Ceramics Center, Nagoya, Japan.
}

Direct imaging of light element atoms inside energy storage materials such as lithium-ion battery materials is becoming indispensable for understanding carrier ion dynamics during battery cell reactions. For this purpose, annular bright filed (ABF) imaging by scanning transmission electron microscopy (STEM) has been widely used [1]. ABF STEM can robustly visualize light element atoms by using an annular detector in the bright field disk region, but cannot be always applicable to very beam-sensitive materials. Although lower accelerating voltage and lower electron dose conditions are necessary to avoid electron irradiation damage, lower accelerating voltage is known to worsen ABF image contrast [2], and lower dose leads to lower signal-to-noise ratio (SNR). As one candidate of high electron-dose efficiency imaging techniques, electron ptychography has been investigated intensively with pixelated detectors in recent years [3]. Ptychography reconstructs phase information efficiently and may be the most effective imaging technique in STEM to observe light element atoms. However, the current pixelated detectors inevitably need long dwell time of electron beam $(\sim 1000 \mu \mathrm{sec} . / \mathrm{pixel})$ and this makes it difficult to achieve low dose imaging conditions in actual experiments. In contrast, segmented detectors with scintillator coupled to photomultipliers enable very fast scan $(\sim 1 \mu \mathrm{sec}$./pixel) and suit for real experimental applications [4]. Therefore, a high contrast light element imaging method with segmented detectors is worthy to be developed for observing beam-sensitive materials.

In this research, the most efficient light-element imaging method has been theoretically designed by the sum of spatial-frequency-filtered images of each annular detector segment. First, integrated phase contrast transfer functions (iPCTFs) [5] are calculated for each detector segment. On the basis of iPCTFs, obtainable signals from thick samples can be estimated at each spatial frequency and in each image within the weak phase object approximation (WPOA). The noise levels in each image can be evaluated based on a recently proposed theoretical framework [6], and thus, SNR can be theoretically maximized at each spatial frequency; namely, spatial filters for each image can be optimized. We call this method optimized-ABF (oABF). Here, the oABF method was applied to simulated and experimental images of $\mathrm{SrTiO}_{3}[001]$ zone-axis.

Figure 1 shows a schematic illustration of the STEM optical system and iPCTFs normalized by the noise-levels of $\mathrm{ABF}$ and $\mathrm{oABF}$. The detector has 4 annular segments and the whole detector area is set to coincide with the bright-field disk. The noise-normalized iPCTFs were calculated with thickness of $15 \mathrm{~nm}$ and in-focus condition, and the profile of oABF shows higher value in the whole spatial frequencies than that of $\mathrm{ABF}$. This result suggests that oABF can decrease electron dose up to about one-tenth compared with ABF. Figure 2 shows simulated and experimental images of ABF and oABF of $\mathrm{SrTiO}_{3}$ [001]. The observation condition is the same as the iPCTFs calculation, and Poisson noise due to the finite electron dose is introduced in the simulated images. Oxygen columns in oABF images are more clearly visualized at lower dose condition than those in ABF images. The experimental images and their intensity profiles also show the better visualization of oxygen atomic columns by oABF. 
References:

[1] S.D. Findlay et al., Applied Physics Letters 95 (2009), 19193.

[2] S.D. Findlay et al., Ultramicroscopy 111 (2011), 1144

[3] J. Lozano et al., Nano Letters 18 (2018), 6850

[4] N. Shibata et al., Journal of Electron Microscopy 59 (2010) 473

[5] T. Seki et al., Ultramicroscopy 194 (2018), 193

[6] T. Seki et al., Ultramicroscopy 193 (2018), 118

[7] The authors acknowledge funding from JST SENTAN, KAKENHI JSPS Grant No. JP17H01316 and Grant-in-Aid for Specially Promoted Research "Atom-by-atom imaging of ion dynamics in nanostructures for materials innovation" (Grant No. JP17H06094).

(a)

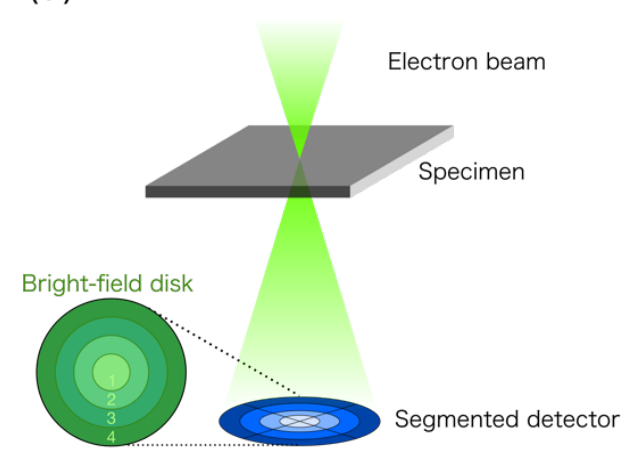

(b)

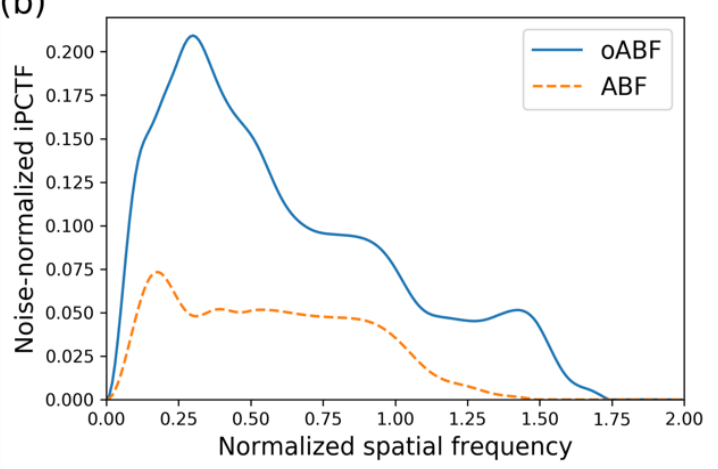

Figure 1. (a) Schematic illustration of STEM optical system with the segmented detector [4]. (b) Noise-normalized iPCTFs of oABF and ABF with thickness of $15 \mathrm{~nm}$, accelerating voltage of $300 \mathrm{kV}$ and convergence semi-angle of $30 \mathrm{mrad}$. As for ABF, the sign of $\mathrm{iPCTF}$ is inverted for comparison.

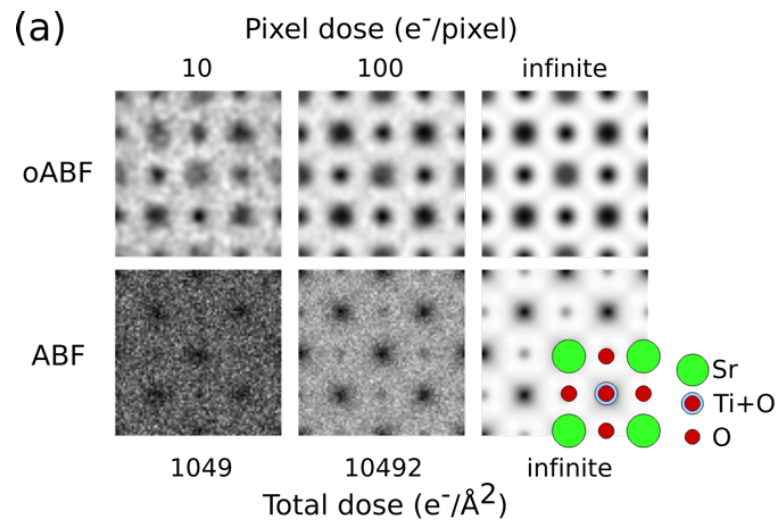

(b)

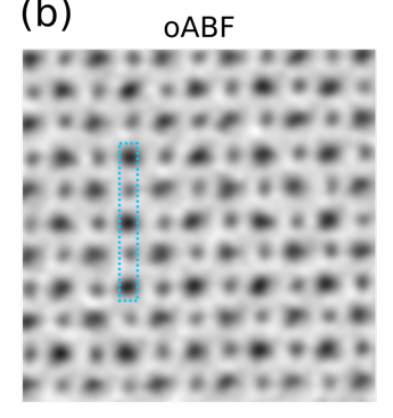

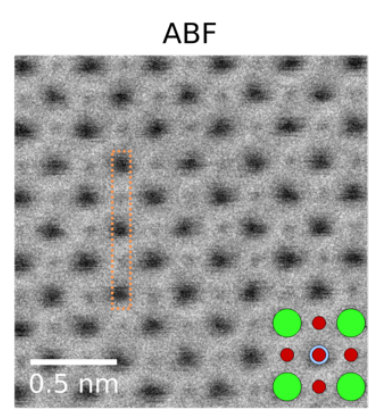

$\mathrm{Sr} O \mathrm{Ti}+\mathrm{O} \bullet \mathrm{O}$
Figure 2. (a) Simulated $\mathrm{OABF}$ and $\mathrm{ABF}$ images of $\mathrm{SrTiO}_{3}$ [001] with the same observation condition as the $\mathrm{PPCTF}$ calculation. The finite electron dose per pixel and $\AA^{2}$ is also shown.

(b) Experimental oABF and $\mathrm{ABF}$ images of $\mathrm{SrTiO}_{3}$ [001] with the same observation condition as the simulations. Intensity profiles obtained from the dashed rectangles' areas in the images are also shown.

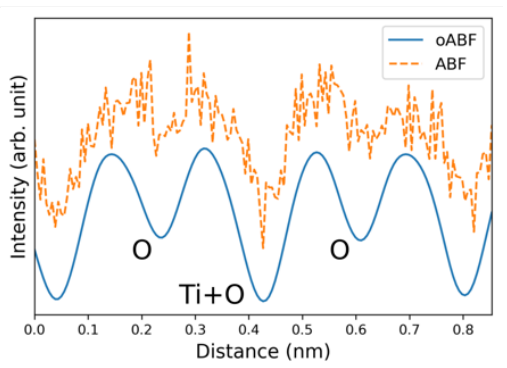

